\title{
Comment lire un texte qui n'existe pas ? Cardenio entre Cervantes et Shakespeare
}

\section{Roger Chartier}

\section{(2) OpenEdition}

\section{Journals}

Édition électronique

URL : http://journals.openedition.org/shakespeare/1924

DOI : 10.4000/shakespeare. 1924

ISSN : 2271-6424

Éditeur

Société Française Shakespeare

Édition imprimée

Date de publication : 1 avril 2013

Pagination : 87-106

ISBN : 2-9521475-9-0

\section{Référence électronique}

Roger Chartier, "Comment lire un texte qui n'existe pas ? Cardenio entre Cervantes et Shakespeare », Actes des congrès de la Société française Shakespeare [En ligne], 30 | 2013, mis en ligne le 03 avril 2013, consulté le 01 mai 2019. URL : http://journals.openedition.org/shakespeare/1924 ; DOI : 10.4000/

shakespeare.1924 


\section{Shakespeare et la mémoire}

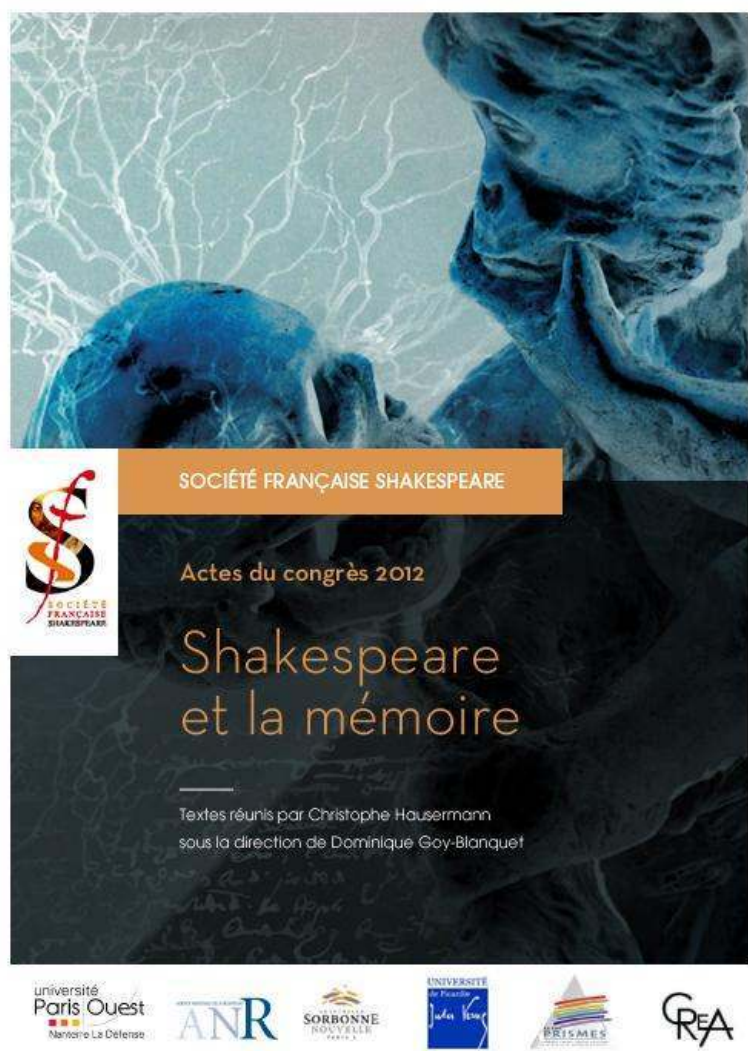

actes du Congrès

organisé par la

SOCIÉTÉ FRANÇAISE SHAKESPEARE

les 22, 23 et 24 mars 2012

textes réunis par

Christophe HAUSERMANN

sous la direction de

Dominique GoY-BLANQUET 


\section{COUVERTURE}

d'après l'affiche de Claire Colombet

conception graphique et logo

Pierre Kapitaniak

mise en page et corrections

Christophe Hausermann

(C) 2012 Société Française Shakespeare

Institut du Monde Anglophone

Université de Paris III - Sorbonne Nouvelle

5 rue de l'École de Médecine 75006 Paris

www.societefrancaiseshakespeare.org

Tous droits de traduction, de reproduction et d'adaptation réservés pour tous les pays 


\title{
COMMENT LIRE UN TEXTE QUI N'EXISTE PAS ? CARDENIO ENTRE CERVANTES ET SHAKESPEARE 1
}

\author{
Roger Chartier
}

\begin{abstract}
Comment lire un texte qui n'existe pas, représenter une pièce dont le manuscrit s'est perdu et dont on ne sait pas avec certitude qui fut son véritable auteur? C'est l'énigme que pose Cardenio - une pièce jouée en Angleterre pour la première fois en 1612 ou 1613 et attribuée quarante ans plus tard à Shakespeare (et Fletcher). Elle a pour trame une «nouvelle » insérée dans Don Quichotte, œuvre qui circula dans les grands pays européens où elle fut traduite et adaptée pour le théâtre. En Angleterre, le roman de Cervantès était connu et cité avant même d'être traduit en 1612 et d'inspirer Cardenio. Retracer l'histoire de cette pièce conduit alors à s'interroger sur ce que fut, dans le passé, le statut des œuvres jugées aujourd'hui canoniques. La " mémoire » de l'histoire de Cardenio est fluctuante, elle varie au gré de la malléabilité des textes, transformés par leurs traductions et leurs adaptations, par leurs migrations d'un genre à l'autre et par les significations successives qu'en construisirent leurs différents publics.
\end{abstract}

How can we read a text that doesn't exist, show a play whose manuscript has been lost and whose author isn't known for sure? This is Cardenio's riddle - a play performed in England for the first time in 1612 or 1613 and attributed forty years later to Shakespeare (and Fletcher). The plot is based on a "short story" inserted in Don Quichotte, a book that circulated in most European countries, where it was translated and adapted for theater performance. In England, Cervantes's novel was known and quoted before being translated in 1612 and therefore before inspiring Cardenio. Tracing back the story of this play makes us question the status of past works which are deemed canonical today. The "memory" of Cardenio's story is changing, it varies according to the mutability of the texts, transformed by their translations and adaptations, by their migrations from one genre to the other and by the successive meanings different audiences have constructed from them.

I

$\mathrm{L}$ 'histoire commence avec un registre de comptes, celui où furent inscrits les paiements faits par le Trésorier de la Chambre du roi d'Angleterre. En date du 20 mai 1613, il mentionne le versement de cent cinquante quatre livres, six shillings et huit pence à John Heminges, l'un des acteurs et propriétaires de la troupe des King's Men, officiellement désignés comme Grooms of the Chamber, pour les représentations de vingt pièces données durant les semaines ou les mois précédents à la cour. Parmi ces vingt pièces, l'une est désignée

\footnotetext{
${ }^{1}$ Ce texte est celui d'une conférence prononcée le 23 mars 2012 dans le cadre du Congrès annuel de la Société Française Shakespeare. Il résume les principales analyses et conclusions de mon livre Cardenio entre Cervantès et Shakespeare. Histoire d'une pièce perdue (Paris, Gallimard, 2011) qui est paru en traduction espagnole (Barcelone, Gedisa) et portugaise (Rio de Janeiro, Record) durant l'année 2012, et en traduction anglaise (Cambridge, Polity Press) en 2013. Nous avons volontairement conservé à cette conférence son caractère oral, ce qui explique l'absence de notes.
} 
sous le titre de Cardenno. Un mois et demi plus tard, le 9 juillet 1613 , la somme de six livres, treize shillings et quatre pence est payée au même John Heminges et à la troupe des comédiens du Roi pour la représentation devant l'ambassadeur du duc de Savoie, hôte du souverain anglais, d'une pièce mentionnée cette fois-ci avec le titre de Cardenna. C'est cette pièce au titre instable, Cardenno ou Cardenna, dont j'ai voulu percer le mystère.

Grâce aux paiements faits à la troupe des King's Men, il est possible de connaître, sinon la date exacte, du moins les circonstances de sa première représentation. La pièce a été l'un des spectacles donnés lors des deux cycles festifs qui, dans toute l'Europe chrétienne, sont des temps de réjouissances et de divertissements : le cycle des douze jours, entre le jour de Noël et l'Epiphanie, désignée en Angleterre comme « Twelfth Night », puis la période de Carnaval, entre le 2 février et le 2 mars. Une intense activité théâtrale, dans les cours comme dans les villes, accompagne les fêtes et les coutumes propres à ces deux moments essentiels du calendrier. En Angleterre, à ces circonstances ordinaires, l'hiver de 1612-1613 en ajoute d'autres, plus exceptionnelles. Le 6 novembre 1612 est mort le fils aîné de Jacques I ${ }^{\text {er }}$, le prince Henry qui sera enterré à Westminster le 7 décembre, et le 14 février 1613, jour de la Saint Valentin, la fille du roi, Elizabeth, a épousé l'Electeur du Palatinat. Les festivités des douze jours et du Carnaval sont donc marquées par la douleur du deuil et la joie de l'hyménée.

Parmi les vingt pièces mentionnées par le registre de comptes, pourquoi s'attacher plus particulièrement à Cardenno ? À l'évidence parce que ce nom renvoie à un livre traduit en anglais en 1612 et publié dans sa langue originale à Madrid en 1605: Don Quichotte de la Manche. Il ne fait pas de doute, en effet, que Cardenno est Cardenio, le jeune noble andalou qui par désespoir d'amour a fait retraite dans la Sierra Morena où il se conduit en homme sauvage, les habits déchirés, le visage brûlé par le soleil, sautant de rocher en rocher. Don Quichotte le rencontre au chapitre XXIII de l'histoire. Les malheurs de Cardenio, amoureux infortuné de Luscinda, trahi par son ami Fernando, et, après de nombreux épisodes, le dénouement finalement heureux, pouvaient fournir une belle matière pour une pièce, à la fois tragédie et comédie, représentée en des jours de peine et de joie à la cour d'Angleterre.

De là, une première et double question : pourquoi le choix de l'histoire écrite par Cervantès pour écrire une pièce de théâtre et 
pourquoi, dans cette histoire du chevalier errant, privilégier les malheurs et bonheurs de Cardenio ? Pour le comprendre, il faut rappeler, tout d'abord, la forte présence espagnole sur les scènes londoniennes. Elle prend différentes formes. La première est la localisation de l'action dramatique en Espagne : ainsi, avec la première et plus fameuse des pièces espagnoles, The Spanish Tragedy de Thomas Kyd. Écrite entre 1582 et 1592, et vraisemblablement après 1585 , la pièce inaugure le genre des "revenge plays", inspiré de Sénèque, et lui donne pour cadre les terres ibériques. Un second motif est la figure de l'Espagnol maniéré et poltron, tel que l'est Don Adriano de Armado, le poète alambiqué, amoureux ridicule et bravache fanfaron de Love's Labour's Lost de Shakespeare, publié en 1598. La figure divertissante et dérisoire de l'extravagant Armado est comme un contrepoint rassurant aux descriptions dénonçant les cruautés infligées aux habitants du Nouveau Monde, rappelées pour mettre en garde contre celles qu'ils pourraient perpétrer dans l'ancien contre les Protestants. C'est ainsi qu'est présentée la traduction, publiée en 1583 sous le titre de The Spanish colonie, de la Brevissima relación de la destruyción de las Indias de Las Casas, imprimée à Séville en 1552 et fondatrice de la légende noire anti-espagnole. Dans la guerre, d'abord, et ensuite dans la paix, signée à Londres en 1604 puis à Valladolid en 1605, la référence espagnole hante l'imagination des auteurs anglais et, parmi eux, les dramaturges.

C'est dans ce contexte qu'est publiée en 1612 la traduction de Don Quichotte par Thomas Shelton. Dès avant sa publication, des allusions à l'histoire du chevalier errant apparaissent dans des pièces de Wilkins, Middleton et Ben Jonson. La plus fameuse est The Knight of the Burning Pestle, représentée en 1607 ou 1611 et attribuée à Beaumont et Fletcher sur la page de titre des éditions de 1635 - mais considérée comme écrite par le seul Beaumont par la plupart des éditeurs modernes. Même s'il faut se garder d'une mise en parallèle trop étroite entre la comédie et l'histoire écrite par Cervantès et même si Beaumont (avec ou sans Fletcher) puise son inspiration directement dans les romans de chevalerie eux-mêmes, et non dans leur parodie, il parait assuré que le dramaturge connaissait les aventures de Don Quichotte. Elles sont le contrepoint de celles de Ralph, le commis d'épicerie qui se fait "grocer errant" sur la scène du Théâtre des Blackfriars pour plaire à son patron et à la femme de celui-ci, lassés 
des satires contre les «citizens» de Londres qui y sont jouées ordinairement.

Comment l'œuvre de Cervantès a-t-elle pu être connue en Angleterre avant la publication de sa traduction imprimée ? On ne peut écarter, tout d'abord, l'hypothèse de sa lecture dans l'une ou l'autre des éditions en castillan publiées avant 1608 : cinq en 1605 (deux à Madrid, deux à Lisbonne, une à Valence), une en 1607 (à Bruxelles), une en 1608 (à Madrid de nouveau). Avant la traduction de Shelton, deux autres éditions du texte de Cervantès sont en circulation : celle de Milan en 1610 et la seconde de Bruxelles en 1611. Avec neuf éditions parues avant 1612 Don Quichotte connait une large circulation, qui ne se limite ni à la péninsule ibérique, ni à l'Amérique espagnole. Il est donc plus que probable que certains lecteurs anglais ont pu acquérir, recevoir et lire Don Quichotte dans sa langue dès 1605 ou 1606, aidés par les nombreux dictionnaires, grammaires et manuels destinés à enseigner l'espagnol publiés par les éditeurs londoniens à partir de 1590. D'autres lecteurs ont pu connaître l'histoire grâce à la circulation manuscrite de la traduction de Shelton, achevée cinq ou six ans avant sa publication imprimée.

Les folies de don Quichotte ont donc été connues très tôt en Angleterre. Mais pourquoi, alors, en 1612 ou 1613, la pièce représentée deux fois par les King's Men fait-elle de Cardenio, et non du chevalier errant son héros principal ? Pourquoi suggère-t-elle par son titre que son intrigue est celle des amours contrariées et finalement satisfaites du jeune noble andalou, et non les aventures comiques de l'hidalgo et son écuyer ? La réponse n'est pas aisée puisque jamais la pièce ne fut publiée et qu'il n'en subsiste aucun manuscrit. Cette situation, au demeurant, n'a rien d'extraordinaire puisque la très grande majorité des pièces représentées en Angleterre entre 1565 (date de l'édition de The Tragedie of Gordobuc) et 1642 (date de la fermeture des théâtres) ne fut jamais imprimée. David Scott Kastan avance l'idée que moins du cinquième le fut alors que Douglas A. Brooks se montre un peu plus généreux et indique, à partir d'une comparaison entre le nombre de titres connus et celui des textes existants, que c'est un peu plus du tiers des pièces représentées qui a eu au moins une édition imprimée. En l'absence du Cardenio de 1613, seule une série d'hypothèses peut rendre compte de la décision qui transforme en une pièce de théâtre 
cette histoire d'amours racontée par plusieurs de ses protagonistes au fil des chapitres de Don Quichotte.

Pour comprendre ce choix, il faut faire retour à l'histoire écrite par Cervantès. Cardenio, le jeune noble andalou, est amoureux de Luscinda qui lui rend son amour. Les deux jeunes gens ont échangé des promesses qui, avant même la cérémonie nuptiale, ont sanctionné leur union. Malheureusement, Cardenio est trahi par son ami Fernando, le fils du duc, qui, après avoir séduit et abandonné Dorotea, avec laquelle il a échangé promesses et serments qui l'engagent, tombe amoureux de Luscinda, obtient sa main du père de celle-ci. Il l'épouse, malgré la résistance de la jeune fille, selon le rituel de l'Église catholique, dans la maison de son père, en présence de Cardenio, caché derrière une tapisserie, témoin muet et impuissant de son infortune. Le mariage n'est pas consommé parce que Fernando a quitté la ville après avoir lu un billet dans lequel Luscinda, qui s'est évanouie durant la cérémonie, déclarait être l'épouse de Cardenio. La jeune fille, elle aussi, a quitté la maison paternelle pour se réfugier dans un couvent.

Ces infortunes, le lecteur de l'histoire les connait car elles sont racontées par Cardenio à don Quichotte lors de leur rencontre dans la Sierra Morena où tous deux ont décidé de faire retraite, comme Amadis ou comme Orlando. Le récit est complété plus loin par Dorotea, partie à la recherche de l'infidèle Fernando, après de nombreux épisodes, qui lient étroitement la «nouvelle» des quatre jeunes gens et les aventures, ou mésaventures du chevalier errant et de son gros écuyer. Finalement, au chapitre XXXvI du livre de Cervantès, tous se retrouvent dans l'auberge de Juan Palomeque. Après reconnaissances, repentirs et pardons, les couples qui s'étaient promis en mariage se reforment. Tous sortent de l'auberge - et de l'histoire - après avoir accompagné un temps don Quichotte, au chapitre XLVII d'un livre dans lequel Cardenio était entré vingt-quatre chapitres plus tôt.

L'histoire narrée par Cardenio pouvait ainsi fournir une bonne intrigue pour le genre tragi-comique alors à la mode. Fletcher l'avait ainsi défini dans l'adresse au lecteur qui précède sa pièce, The Faithful Shepherdess, en 1608: "A tragi-comedy is not so called in respect of mirth and killing, but in respect it wants deaths, which is enough to make it no tragedy, yet brings some near it, which is enough to make it no comedy, which must be a representation of familiar people, with such kind of trouble as no life be questioned ». Les amours des jeunes 
gens entraient tout à fait dans cette définition puisque Cardenio, Luscinda et Dorotea, chacun à son tour, songent au suicide ou frôlent la mort, mais personne ne meurt et tout est bien qui finit bien.

Nous ne saurons sans doute jamais comment « ces aventures si enchevêtrées et si désespérées », comme écrit Cervantès, furent portées sur la scène par les Comédiens du roi lorsqu'en 1612 ou 1613, par deux fois, ils représentèrent Cardenio. Si le texte de Cervantès et la traduction de Shelton, qui le suit fidèlement, proposaient des matériaux immédiatement utilisables pour une pièce de théâtre, avec des moments spectaculaires (le mariage, la séduction, les reconnaissances, les adieux), des dialogues dramatiques et des monologues intérieurs, il n'en allait pas de même avec la construction même de l'intrigue. Comment, en effet, transformer en un récit linéaire ce qui était donné dans Don Quichotte comme une série de retours en arrière où chaque narration ajoutait des épisodes connus seulement par celui ou celle qui convoquait le passé dans sa mémoire? Et, plus difficile encore, comment traiter sur le théâtre l'intrication de la « nouvelle » de Cardenio dans l' « histoire » de don Quichotte ? L'enjeu n'était pas mince car il pouvait conduire soit à représenter les amours de Cardenio et Fernando sans les lier d'aucune manière aux aventures de don Quichotte, soit à inventer une formule qui associait sur la scène la déraison comique du chevalier errant et la nouvelle sentimentale des amants séparés puis réunis. Une pièce fondée sur Don Quichotte pouvait-elle ignorer son héros principal ? Ou bien devait-elle, comme l'histoire parue en 1605, jouer des multiples effets que produit la rencontre entre les folies de don Quichotte et celles de Cardenio ? En l'absence du texte, il est impossible de le dire.

Ce qui est sûr, en revanche, c'est que, dès les premiers temps de sa circulation, Don Quichotte est apparu non seulement comme la parodie comique des romans de chevalerie (et d'autres genres comme le roman picaresque et la fable pastorale), mais aussi comme une anthologie de «nouvelles » qui pouvaient fournir aux dramaturges une matière riche en coups de théâtre, en scènes dramatiques, en sentiments violents et contrastés. Le livre offrait, en effet, de nombreuses possibilités avec ses « novelas » emboîtées dans l'histoire.

Comme le rappelle Samson Carrasco au début de la seconde partie de Don Quichotte parue en 1615 , le reproche fut adressé à Cervantès d'avoir intercalé dans l'histoire du chevalier errant une 
« novela » qui n'avait rien à voir avec l'histoire de don Quichotte : la nouvelle du Curieux impertinent qui occupe les chapitres XXXIII à XXXV. C'est, en effet, un récit dans le récit, lu à haute voix par le curé aux autres personnages. Son adaptation théâtrale était donc aisée. Sans doute écrit entre 1608 et 1610, The Coxcomb de Beaumont et Fletcher reprend la situation de la nouvelle, Antonio incitant avec insistance son ami Mercury à courtiser sa propre femme. En 1611, une pièce de Thomas Middleton, The Second Maiden's Tragedy, porte sur la scène comme intrigue secondaire l'histoire d'Anselmo, le mari trop curieux ou trop sûr de la vertu de sa femme, et de son ami Lotario (devenu Votarius), mis au défi de conquérir celle-ci et pris au jeu de la séduction. Plus tard, The Renegade, or, The Gentleman of Venice, une pièce de Philip Massinger autorisée en 1624 et publiée en 1629, fut sans doute inspirée par une autre «nouvelle » inscrite dans l'histoire : les chapitres XXXIX à XLI où Ruy Pérez de Viedma, le capitaine capturé par les Barbaresques et échappé des prisons d'Alger, raconte son aventure. Les amours de Cardenio présentaient de plus grandes difficultés puisque, dans ce cas, la «nouvelle» se trouve fortement et durablement liée aux pérégrinations du chevalier errant. En 1613, c'est pourtant cette première histoire dans l'histoire qui fut représentée par les King's Men. L'argent fut versé à John Heminges, l'un des acteurs et propriétaires, ou "shareholders » de la compagnie, et non pas à l'auteur, ou aux auteurs, jamais nommés dans les comptes du trésorier de la Chambre du Roi.

\section{II}

Ils ne le seront que quarante ans plus tard. Le 9 septembre 1653 le libraire Humphrey Moseley fait enregistrer par la communauté des libraires et imprimeurs londoniens, la Stationers' Company, les titres de quarante et une pièces de théâtre sur lesquelles il possède dès lors un " right in copy », c'est-à-dire un droit de propriété perpétuel et exclusif. Il lui en coûte vingt shillings et six pence qui lui assurent, selon les règles de la communauté, le monopole de l'impression des œuvres qu'il a ainsi « entered », fait enregistrer. Parmi ces quarante et une pièces, quatre sont attribuées à Shakespeare: «Henry ye. first, \& Hen: ye 2d. by Shakespeare, \& Davenport ", "The merry Devill of Edmonton. By Wm: Shakespeare », et « The History of Cardenio, by 
Mr Fletcher. \& Shakespeare ». Des deux Henry on ne sait rien, sinon qu'une pièce intitulée The History of Henry the First avait été autorisée en 1624 et attribuée alors à Davenport. The Merry Devill of Edmonton a pour sa part été enregistrée en 1607 et publiée quatre fois entre 1608 et 1653. Reste The History of Cardenio qui est sans doute la pièce jouée quarante ans plus tôt devant la cour et dont, pour la première fois, les auteurs sont nommés : Fletcher et Shakespeare.

La mention du registre des libraires et imprimeurs de Londres n'est pas sans laisser quelque incertitude. Tout d'abord, le nom de Shakespeare, écrit après un point, pourrait paraître comme ajouté après coup. Ensuite, les attributions de Moseley sont souvent fantaisistes. C'est ainsi que dans une autre «entrée », daté de 1660, il porte au crédit de Shakespeare des pièces qu'aucune révision du canon ne lui attribuera : « The History of King Stephen », « Duke Humphrey. a Tragedy» et «Iphis \& Iantha, Or a marriage without a man. a Comedy ». Le doute est donc permis quant à la parfaite exactitude du document de 1653, le premier et le seul à lier Shakespeare et Cardenio au XVII ${ }^{e}$ siècle. Une collaboration entre Fletcher et Shakespeare en 1613 est, toutefois, tout à fait vraisemblable puisque entre 1612 et 1614 ils composèrent ensemble deux autres pièces: d'abord, All is True, devenue dans le Folio de 1623 The Life of King Henry the Eight, et The Two Noble Kinsmen.

Le sort des trois pièces fut fort différent. Dans le Folio de 1623, Heminges et Condell inclurent Henry VIII et exclurent The Two Noble Kinsmen, fidèles à leur projet qui entendait réunir, pour leur édition des Comedies, Histories, \& Tragedies de Shakespeare, «his owne writings ", ses propres écrits, comme ils le disent dans leur adresse aux lecteurs. Ils ont donc écarté les pièces dont ils savaient ou supposaient qu'elles avaient été écrites en collaboration (ce qui, pour eux, n'était pas le cas de Henry VIII dont l'attribution au seul Shakespeare n'a été mise en doute qu'au XIXe siècle). Si les éditeurs du Folio de 1623 possédaient un manuscrit de The History of Cardenio, ils l'ont traité comme The Two Noble Kinsmen, et non comme All is True : ils l'ont donc exclu. La pièce ne sera jamais publiée, ni dans les rééditions du Folio shakespearien de 1623, pas même dans celles de 1664 et 1685 qui ajoutent sept pièces au corpus canonique des trente-six pièces réunies dans le premier Folio, ni dans le Folio de 1647 qui rassemble trente- 
cinq pièces de Fletcher et Beaumont et dont l'éditeur est pourtant Moseley lui-même.

En 1653, l'enregistrement d'une "copy " de The History of Cardenio doit être situé au sein de la politique éditoriale de Humphrey Moseley, membre de la Stationers' Company depuis 1633 et fervent royaliste. Pour lui, faire enregistrer par la Stationers' Company un très grand nombre de pièces de théâtre du temps d'Elizabeth et des premiers Stuarts avait une signification politique puisque, depuis 1642, les théâtres étaient fermés et les représentations publiques interdites. Dans cette perspective, The History of Cardenio enregistrée en 1653 n'avait pas une particulière importance, si ce n'est qu'avec trois autres titres (les deux parties de Henry the First et The Merry Devill of Edmonton), elle aurait été composée, au moins en partie, par l'un des quatre dramaturges dont les œuvres avaient été rassemblées dans le prestigieux format du Folio, après les Workes de Ben Jonson en 1616 et avant les pièces de Beaumont et Fletcher rassemblées en en 1647.

Mais, le « right in copy » acquis par Moseley sur une pièce inspirée par Don Quichotte peut être également situé dans un autre contexte : le « revival » de l'histoire de Cervantès à la mi-XviI siècle en Angleterre. Il se marque, d'abord, par l'entrée de nouveaux mots dans le vocabulaire. Dès 1642, l'adjectif "quixotical » avait ouvert cette première série de termes, construit à partir du nom du héros de Cervantès dans un pamphlet de John Taylor, le prolifique « water poet ». Il est suivi en 1644 par l'emploi de «Quixote » comme un nom commun désignant des individus aussi fous ou déraisonnables que le chevalier errant, puis par le verbe « to be Don Quixoted » qui apparait en 1648.

Multiples sont les références au chevalier errant, mises au service des factions en conflit, dans les gazettes et les pamphlets. Durant la guerre civile, don Quichotte ne semble pas avoir de parti : défenseurs zélés du Parlement et royalistes militants convoquent les uns et les autres la figure chimérique du chevalier errant pour ridiculiser leurs adversaires et montrer que leur propre cause est celle du bon sens et de la raison. Cardenio et don Quichotte sont donc très présents dans l'Angleterre de la mi-XVII siècle. Moseley aurait pu ou aurait dû en profiter pour publier The History of Cardenio, un texte dont il était propriétaire depuis le 9 septembre 1653. Il ne l'a pas fait, laissant entier le mystère de ce titre sans texte, sinon sans auteurs. 
L'histoire aurait pu en rester là - et également cette conférence - si en 1727 Lewis Theobald, l'un des trois premiers éditeurs des œuvres de Shakespeare au XVIII ${ }^{\mathrm{e}}$ siècle, après Nicholas Rowe et Alexander Pope, n'avait pas fait représenter sur la scène du Theatre Royal à Drury Lane à Londres une pièce intitulée Double Falshood, or The Distrest Lovers. La pièce fut publiée l'année suivante, avec une page de titre qui faisait mention de la destructrice critique de l'édition de Pope par Lewis Theobald, publiée trois ans auparavant. Dans sa préface, Theobald affirmait posséder plusieurs manuscrits d'une pièce perdue de Shakespeare. Le titre de 1653, The History of Cardenio, n'est pas mentionné, mais les spectateurs et les lecteurs de sa pièce n'avaient aucune peine à reconnaître les personnages de la « nouvelle » imaginée par Cervantès sous leurs nouveaux noms: Julio pour Cardenio, Leonora pour Luscinda, Violante pour Dorotea et Henriquez pour Fernando. Double Falshood est bien l'histoire de Cardenio/Julio trahi par le perfide Fernando/Henriquez, tout comme l'a été Dorotea/Violante, séduite puis abandonnée par le fils du duc. Mais à la différence de Cervantès, Lewis Theobald n'associait pas les mésaventures des amants affligés et finalement réunis avec les aventures de don Quichotte et Sancho. Ceux-ci n'apparaissent pas dans sa pièce et aucune n'allusion n'est faite au chevalier errant. Faut-il en conclure qu'il en allait déjà ainsi en 1612-1613 et que Fletcher et Shakespeare avaient pris le même parti ? Ce serait sans doute oublier que leur pièce a été " revised » et «adapted » avec la liberté que s'autorisaient les dramaturges du XVIII ${ }^{\mathrm{e}}$ siècle lorsqu'ils s'emparaient des œuvres anciennes.

Si Theobald dit vrai, sa pièce serait la seule trace textuelle survivant du Cardenio cent vingt cinq ans auparavant. Elle pose deux questions fondamentales. Celle de la relation "schizophrénique ", comme a pu l'écrire David Scott Kastan, des éditeurs et dramaturges anglais du XVIII ${ }^{\mathrm{e}}$ siècle avec Shakespeare, restauré et altéré, édité avec érudition et adapté avec une totale liberté. Celle de l'omniprésence de Don Quichotte dans l'Angleterre de la Restauration et de la première moitié du XVIII ${ }^{\mathrm{e}}$ siècle, attestée par les traductions, les abrégés et les adaptations théâtrales de l'histoire écrite par Cervantès. 
L'étude de la pièce de Theobald permet de repérer le travail d'adaptation qu'il a opéré sur le texte de Cervantès, lu dans l'original espagnol ou dans la traduction de Shelton de 1612. Au-delà de la redistribution chronologique des événements toujours relatés rétrospectivement dans la "nouvelle », son intrigue présente deux éléments étrangers au récit de 1605 : d'une part, l'accent mis sur les relations entre les pères et leurs enfants, ce qui situe les amours et désamours des quatre amoureux à l'intérieur d'une comédie domestique où entrent en conflit la nécessaire obéissance des jeunes gens et leur droit légitime à choisir ; d'autre part, le rôle d'arbitre des tensions attribué au duc et à son fils aîné, grâce auxquels la vérité se révèle, le méchant est contraint au repentir et les heureux desseins de la Providence se réalisent.

Faut-il imputer ces transformations à la révision de Theobald, aux auteurs de 1612-1613 ou bien, à une possible adaptation des années 1660 puisque l'un des manuscrits que Theobald disait posséder était de la main de Mr. Downes, l'ancien régisseur de Davenant, et il aurait été possédé par Thomas Betterton, l'un des plus fameux acteurs de cette troupe qui est l'une des deux à reprendre les représentations de théâtre après la Restauration ? Il est difficile de le dire. D'un côté, se rencontrent dans la pièce de Theobald des thèmes rencontrés également dans Henry VIII et The Two Noble Kinsmen, les deux autres pièces issues de la collaboration entre Fletcher et Shakespeare entre 1612 et 1614, par exemple, celui de l'amitié brisée entre deux jeunes hommes par l'amour ancien ou soudain qu'ils portent à la même femme. D'un autre côté, la comédie familiale qui oppose puis réunit les pères et leurs fils ou leurs filles est une trame familière au théâtre anglais de la Restauration et du premier XVIII ${ }^{\mathrm{e}}$ siècle.

Demeure une question fondamentale : à quelle réécriture (1613, 1660, 1727) faut-il attribuer l'absence de don Quichotte et Sancho dans la pièce de 1727 ? Si l'on suppose que don Quichotte était déjà absent de la History of Cardenio en 1613, deux raisons peuvent être invoquées : d'une part, l'échec sur le théâtre de la première comédie inspirée par les exploits du chevalier errant, The Knight of the Burning Pestle; d'autre part, la renaissance de l'idéal de la croisade entretenue par le prince Henry, mort en 1612, fort peu favorable à la satire de la chevalerie. Quoi qu'il en soit, l'absence du héros de l'histoire dans la pièce anglaise prive le spectateur et le lecteur des extravagances de don 
Quichotte et de ses rencontres avec Cardenio. Ce parti n'avait pas été celui adopté par d'autres dramaturges qui, avant ou après Fletcher et Shakespeare, avaient porté sur le théâtre les amours de Cardenio, mais sans exclure le chevalier errant de leur pièce. Il en allait ainsi du Don Quijote de la Mancha, une « comedia » du Valencien Guillén de Castro, sans doute écrite avant 1608 , ou, à Paris, d'une tragi-comédie intitulée Les Folies de Cardenio composée par Pichou et représentée 1628. Theobald connaissait-il leurs pièces et voulut-il s'en écarter en dénouant le lien qu'elles avaient serré entre le chevalier errant et les jeunes amoureux ? La question reste sans réponse, créant une autre incertitude dans cette histoire d'une pièce disparue.

$\mathrm{Au} \mathrm{Xx}^{\mathrm{e}}$ siècle, les affirmations de Theobald quant à l'authenticité shakespearienne de Double Falshood ont obsédé les critiques en quête du Cardenio perdu. Commençons par la plus essentielle. Faut-il le croire et accepter que Double Falshood est bien une adaptation d'une pièce du XvII ${ }^{e}$ siècle dont il possédait un ou plusieurs manuscrits ? Ou bien doit-on tenir Double Falshood comme une complète mystification dont il serait l'unique auteur? La thèse de la falsification repose sur deux éléments essentiels. D'une part, du fait de sa profonde connaissance de Shakespeare, à la fois comme critique (dans son éphémère périodique The Censor), comme poète (imitant Shakespeare dans The Cave of Poverty), comme dramaturge (en 1720 il compose un Richard II «alter'd from Shakespeare ») et comme éditeur, Theobald était tout à fait capable d'imiter l'écriture shakespearienne. D'autre part, sa familiarité avec Don Quichotte lui permettait de trouver dans l'histoire écrite par Cervantès des intrigues de théâtre - à preuve, en 1741, quinze ans après Double Falshood, son « English opera » intitulé The Happy Captive, dont la source est le récit du captif évadé des prisons d'Alger avec la belle et chrétienne Zoraida.

Séduisante, la thèse se heurte, toutefois, à de fortes objections. Il paraît, en effet, peu probable que Theobald ait pris le risque d'une telle supercherie au moment même où il entendait établir son autorité comme éditeur de Shakespeare en corrigeant les erreurs de Pope dans Shakespeare Restor'd et, ainsi, apparaître comme le seul savant véritablement qualifié pour une nouvelle édition des œuvres du « Poète national». Par ailleurs, un risque plus grand encore aurait été de compromettre l'autorité du dédicataire de l'édition de la pièce en 1728, Sir George Dodington, et celle du souverain lui-même, avec 
l'inhabituelle présence des armes royales et du texte entier du privilège dans cette même édition. Il est donc peu vraisemblable que Theobald ait voulu assumer de tels risques pour le seul plaisir ou profit d'une mystification.

D'autres arguments, fondés sur l'étude de la pièce elle-même, peuvent être avancés pour conforter l'idée que Double Falshood est bien une adaptation d'une pièce antérieure dont les auteurs sont bien Fletcher et Shakespeare : ainsi, la présence dans Double Falshood de formulations très proches, par leur vocabulaire ou leur structure, de passages qui n'ont de parallèles que dans des œuvres de ceux-ci, ou bien la présence de deux styles poétiques dans la pièce, reconnaissables à partir de la distribution inégale dans la pièce des «feminine endings » des vers, une forme poétique caractéristique de l'écriture de Fletcher, et non de celle de Shakespeare. Ainsi, si Theobald a voulu produire un faux shakespearien, il aurait contrefait dans une grande partie de la pièce un autre auteur. Tout laisse donc à penser que, même s'il n'a pas eu connaissance de l'attribution de 1653 , il a pris conscience que la pièce dont il composait une adaptation avait été écrite en collaboration. La «relique» shakespearienne qu'il disait avoir exhumée, et que le public de 1727 attendait avec émotion et révérence, n'était peut-être pas aussi authentique qu'il l'aurait voulu. C'est sans doute pour cela qu'il laissa son Cardenio alias Double Falshood en dehors de son édition des œuvres de Shakespeare qu'il publia en 1733.

IV

Quand j'ai commencé cette recherche, l'énigme de Cardenio n'était discutée qu'entre les érudits qui acceptaient ou refusaient de déceler dans Double Falshood les traces ou les restes d'une pièce écrite par Shakespeare. Il n'en va plus de même aujourd'hui. Depuis quelques années, en effet, une véritable fièvre « cardeniesque » s'est emparée de l'Angleterre et des Etats-Unis. Le mystère de la pièce disparue a séduit plusieurs romanciers qui l'ont transformé en enquêtes policières et " detective stories ». Le premier fut en 2002 Jesper Fford. Dans Lost in a Good Book, son héroïne, Thursday Next, agent de la Brigade Littéraire au sein du Service des Opérations Spéciales, rencontre le manuscrit de Cardenio dans la bibliothèque de Lord Volescamper. Il y a été déposé par le politicien Yorrick Kaine, qui l’a dérobé à la « Grande 
Bibliothèque » et qui espère séduire le « vote shakespearien » lors des prochaines élections en rendant au public ce trésor perdu. En 2007, dans Interred with their Bones de Jennifer Lee Carrell, c'est dans une caverne de l'Arizona, sur la tombe d'un cadavre sans pierre tombale, que Kate découvre le manuscrit de Cardenio, conservé dans une sacoche de selle avec un exemplaire de Don Quichotte en espagnol.

Le thème du manuscrit disparu et retrouvé, oublié puis redécouvert, est aussi ancien que la fiction. Rien d'étonnant, donc, à ce que l'imagination de romanciers contemporains ait été séduite par les mystères d'une histoire shakespearienne perdue. Plus surprenante et plus spectaculaire est, depuis une quinzaine d'années, avant même son appropriation par les romanciers, la présence de Cardenio sur les scènes de théatre anglaises et américaines. Il ne va pas de soi, en effet, de jouer une pièce qui n'existe pas - ou plus. Pour le faire, dramaturges et metteurs en scène ont mobilisé plusieurs possibilités. La première consiste à considérer que Cardenio n'est pas une pièce perdue. Son texte a survécu, mais sous un autre titre qui lui fut donné par erreur. C'est le parti choisi lorsque Cardenio fut représenté en février 1995 au Palm Beach Shakespeare Festival, puis repris en mars 1996 au Linhart Theatre de New York. Le miracle littéraire était rendu possible par la publication un an auparavant d'un livre de Charles Hamilton qui identifiait Cardenio avec le manuscrit d'une pièce conservée à laquelle le censeur, Sir George Buc, le «master of revels », aurait attribué le titre de The Second Maiden's Tragedy en l'absence de tout titre sur le manuscrit.

La thèse de Charles Hamilton est loin d'avoir convaincu la critique. S'il est certain, comme on l'a dit, que l'intrigue secondaire de The Second Maiden's Tragedy est bien fondée sur trois chapitres de Don Quichotte, ceux où est lue l'histoire tragique du Curieux impertinent, il est impossible de reconnaitre dans l'intrigue principale, celle du Tyran qui désire la femme aimée de Govianus, légitime héritier dépossédé de son trône, quelque rapport que ce soit avec la « nouvelle» de Cardenio telle qu'elle est contée dans Don Quichotte. Pourtant, sur les scènes anglaises et plus encore américaines, la pièce considérée comme le Cardenio retrouvé de Shakespeare a fait une belle carrière.

Identifier une pièce existante comme étant le Cardenio disparu de Shakespeare n'est pas la seule solution pour qui veut le monter. Il 
est une autre ressource: la pièce de Theobald, Double Falshood, présentée par son auteur comme une « révision » et "adaptation » de l'œuvre de 1613. C'est ce parti qu'a suivi Gary Taylor dans son History of Cardenio, représenté en 2009 à Wellington et, dans une version révisée, en avril 2012 à Indianapolis. Son texte est une « reconstruction » de la pièce de 1613 , lue comme en filigrane dans celle de 1728. Il est présenté comme une " expérimentation » fondée sur une forme d'imagination philologique, le propos étant de retourner au texte premier, celui de Shakespeare et Fletcher, en soumettant le texte de Theobald à un travail semblable à celui « des artistes qui ont restauré la Chapelle Sixtine ou d'autres tableaux ». Il s'agit donc de "désécrire» ou réécrire Double Falshood dans le style des deux dramaturges et, ainsi, de reconstruire le texte perdu. Gary Taylor, convaincu qu'il en allait ainsi en 1613, a réintroduit don Quichotte et Sancho dans la pièce, comme si les deux dramaturges du XVII ${ }^{\mathrm{e}}$ siècle n'avaient pu ignorer dans leur Cardenio les aventures du chevalier errant et son écuyer et comme si leur absence dans Double Falshood était due à Theobald qui aurait retranché une intrigue qu'il tenait comme sans rapport avec celle qui contait les heurs et malheurs des jeunes amoureux.

Une troisième solution consiste à écrire à un Cardenio mais sans prétendre qu'il est celui de 1612 ou 1613. C'est celle qu'ont adoptée Stephen Greenblatt et Charles Mee dans leur Cardenio représenté en 2008 à Cambridge. Leur dessein n'était pas de reconstruire le Cardenio disparu mais de proposer une expérience de " recycling », de réemploi d'une histoire, ou de plusieurs histoires, dans une forme nouvelle, ouverte elle-même aux appropriations - et la pièce de Greenblatt et Mee a déjà été jouée dans des adaptations en espagnol, croate, japonais ou bengali. Elle se veut un exemple de « mobilité culturelle ». La pièce inverse avec une ingéniosité toute shakespearienne la structure narrative de Don Quichotte. L'histoire de Cardenio y est, en effet, présentée comme une pièce de Shakespeare récemment retrouvée et apportée comme un présent de mariage par deux comédiens, alors que l'intrigue principale est empruntée à la nouvelle du Curieux impertinent.

Le dernier Cardenio, pour le moment du moins, est celui qui a été joué par la Royal Shakespeare Company entre avril et octobre 2011 pour la réouverture du théâtre du Swan et le cinquantième anniversaire 
de la troupe. Mise en scène par Gregory Doran, il entremêle des éléments puisés dans le texte original de Cervantès, la traduction de Shelton et l'adaptation de Theobald. La pièce, tout comme celle de 1727, n’invite pas don Quichotte sur la scène, mais donne seulement à voir et entendre l'histoire des amants désunis puis réunis.

La fièvre contemporaine des Cardenios désigne l'une des tensions essentielles qui traversent la culture écrite de l'Europe moderne. D'un côté, elle montre l'extrême mobilité et instabilité des textes, constamment révisés, adaptés, réécrits. Dans le cas de Cardenio, l'absence de tout «Ur-text» abolit les contraintes qui limitent ordinairement les variations textuelles ou les incarnations matérielles de la « même » œuvre. Cardenio est ainsi un laboratoire unique pour de multiples expérimentations textuelles: changer le titre et l'attribution d'une pièce existante sans changer son texte, reconstruire un texte du Xvir ${ }^{e}$ siècle en réécrivant une œuvre du XviII ${ }^{e}$ siècle, ou proposer une réinvention contemporaine d'une histoire ainsi recyclée une nouvelle fois.

Mais, d'un autre côté, la multiplication textuelle des Cardenios témoigne aussi pour une fondamentale stabilité : celle du nom d'auteur et de la reconnaissance canonique. Tous, en effet, revendiquent leur relation avec l'auteur premier : Shakespeare, auquel la pièce de 1612 ou 1613 a été attribuée, mais seulement en partage et comme second nommé, par Humphrey Moseley en 1653. L'obsession pour Cardenio est ainsi hantée par le fantôme de Shakespeare, même si la pièce de 1613 a été écrite en collaboration avec Fletcher, qui en fut peut-être l'auteur principal, même si le texte de Shakespeare n'est lisible que dans le filigrane de sa réécriture par Lewis Theobald. L'histoire du Cardenio perdu fascine comme toutes celles des ouvres dont la disparition crée un manque intolérable. Entre le $\mathrm{XvIII}^{\mathrm{e}}$ siècle et aujourd'hui, le désir de donner un texte et un corps à ce fantôme a inspiré les critiques shakespeariens, les hommes de théâtre et les éditeurs de textes.

Mais ce texte à jamais absent et, pourtant, si souvent arraché à l'oubli porte, peut-être, un enseignement plus général. Il tient à la discordance existant entre le temps de la composition du premier 
Cardenio, celui joué à la cour d'Angleterre en 1612 ou 1613, fondé sur un livre paru en 1605 , et le temps de sa première résurrection, sous le titre de Double Falshood, sur la scène londonienne du Theatre Royal, en 1727. L'adaptation de Theobald n'existe, en effet, que parce qu'elle affirme sa fidélité à Shakespeare. Même si cette attribution a pu être contestée par les incrédules, même si Theobald lui-même en douta suffisamment pour écarter la pièce de sa propre édition de Shakespeare, c'est bien parce qu'elle était présentée comme une relique shakespearienne que sa pièce fut écrite et montée.

En 1727, elle s'inscrit ainsi dans un ordre des discours naissant, qui se fonde sur l'individualisation de l'écriture, l'originalité des œuvres et la canonisation de l'auteur. Certes, l'articulation de ces trois notions, décisive pour la définition de la propriété littéraire, ne trouvera une forme achevée qu'à la fin du XviII ${ }^{\mathrm{e}}$ siècle, à l'époque du sacre de l'écrivain, de la fétichisation du manuscrit autographe et de l'obsession pour la main de l'auteur, devenue garante de l'authenticité de l'œuvre. Il n'en va pas encore ainsi dans l'Angleterre des premières décennies du siècle. Toutefois, le Statut de la reine Anne de 1710, en limitant le « copyright » des libraires sur les textes qu'ils éditaient, a déjà obligé leurs avocats à fonder la propriété littéraire sur l'irréductible singularité de l'écriture. La controverse à propos de l'authenticité shakespearienne de Double Falshood ne peut être séparée de cette mutation de l'ordre du discours qui célèbre les grands auteurs et canonise leur œuvre.

Dans les critiques des détracteurs de Theobald comme dans ses propres doutes resurgissent, pourtant, les fortes traces d'une autre économie de l'écriture, celle qui avait régi la composition de la pièce supposément adaptée. Elle reposait sur de tout autres pratiques: l'écriture en collaboration, exigée par les protecteurs, les troupes ou les entrepreneurs de théâtre; le réemploi d'histoires déjà racontées, de lieux communs partagés, de formules répétées, ou encore, les continuelles révisions ou continuations d'œuvres toujours ouvertes. C'est avec cette manière d'écrire les fictions que Shakespeare a composé ses pièces et c'est avec et contre elle que Cervantès a écrit Don Quichotte. L'indiquer n'est pas oublier que, pour l'un et l'autre, commence très tôt la canonisation de l'écrivain qui fait de son œuvre un monument. Mais ce processus va de pair jusqu'au XvIII ${ }^{\mathrm{e}}$ siècle avec la forte conscience de la dimension collective de toutes les productions 
textuelles (et pas seulement théâtrales) et avec la faible reconnaissance de l'écrivain comme tel. Ses manuscrits ne méritent pas conservation, ses œuvres ne sont pas sa propriété, mais celle des libraires éditeurs, et ses expériences ne nourrissent aucune biographie littéraire, mais tout au plus des recueils d'anecdotes. Il en ira autrement lorsque l'affirmation de l'originalité créatrice entrelacera l'existence et l'écriture, situera les œuvres dans la vie et reconnaîtra celle-ci dans celles-là.

La relation si problématique entre Double Falshood et The History of Cardenio n'est donc pas seulement une histoire de falsification, d'abrégement ou de réécriture. Son enjeu est plus fondamental puisqu'il porte sur la notion même de « littérature » telle que nous la manions presque sans y penser. Son usage rétrospectif, inévitable car il nous faut penser et écrire avec les mots hérités, risque sans cesse l'anachronisme. Quand la littérature n'est pas encore identifiée aux belles-lettres, le terme ne désigne aucunement les œuvres que nous tenons pour « littéraires », mais, au Moyen Age, les textes écrits en latin et au XVII ${ }^{\mathrm{e}}$ siècle, les ouvrages d'érudition. À ce premier écart, lexical, s'en ajoute un second, conceptuel. Sans la dater très précisément, Foucault l'avait désigné en énonçant les traits propres de la «fonction auteur » qui assigne l'unité et la cohérence d'une œuvre à la singularité d'un sujet propriétaire et responsable de ses écrits. Une telle assignation n'est ni universelle ni invariante. Elle caractérise un ordre des discours littéraires qui naît au XVIII ${ }^{\mathrm{e}}$ siècle, plus tôt en Angleterre, plus tard sur le continent. Sa puissance est telle qu'il soumet à ses principes des textes composés antérieurement et reçus avec des attentes et des catégories très différentes. La tension entre la relique sacrée que Theobald prétend offrir à la dévotion du public en 1727 et la pièce de 1612-1613 qui fut représentée seulement deux fois et qui ne fut jamais imprimée, est exemplaire d'une telle discontinuité. 
Même si elles ne peuvent se libérer des mots qui sont les nôtres, nos lectures des fictions composées avant l'invention de la "littérature » doivent faire effort pour reconnaître leur distante étrangeté. C'est là une condition première pour que, paradoxalement peut-être, nous soit compréhensible, délectable ou émouvante leur présence perpétuée.

Roger Chartier Collège de France 